\title{
Thermal abundance of non-relativistic relics with Sommerfeld enhancement
}

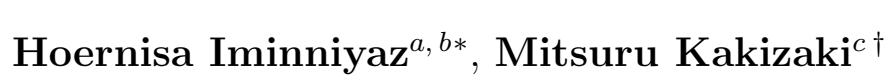 \\ ${ }^{a}$ Center for High Energy Physics, Peking University, Peking, 100871, China \\ ${ }^{b}$ School of Physics Science and Technology, Xinjiang University, Urumqi, 830046, \\ China \\ ${ }^{c}$ LAPTH, Université de Savoie, CNRS, B.P. 110, F-74941 Annecy-le-Vieux Cedex, \\ France
}

\begin{abstract}
We propose an analytic treatment for computing the relic abundances of non-relativistic particles whose annihilation rate at chemical decoupling is increased by Sommerfeld enhancement. We find approximate rational functions that closely fit the thermal average of Sommerfeld-enhanced cross sections in the massless limit of force carriers for $s$ - and $p$-wave annihilations. We demonstrate that, with the approximate thermally-averaged cross sections implemented, the standard analytic method for the final relic abundances provides accuracy to within $1 \%$ even for the case of Sommerfeld enhancement.
\end{abstract}

*wrns@xju.edu.cn

${ }^{\dagger}$ kakizaki@lapp.in2p3.fr 


\section{Introduction}

The determination of the relic abundance of particles which decouple from thermal equilibrium in the early universe is indispensable for understanding the history of the universe. Important examples include the computation of the cosmological dark matter abundance, which provides us a crucial hint for screening dark matter candidates and models beyond the standard model (SM) of particle physics, as well as cosmological scenarios in the early universe. Among many particles proposed, stable or long-lived weakly interacting massive particles (WIMPs) with weak-scale mass are excellent candidates because, assuming the thermal production scenario, the predicted relic density coincides with the dark matter density [1, 2]. The value extracted from the Wilkinson Microwave Anisotropy Probe (WMAP) data is [3],

$$
\Omega_{\mathrm{DM}} h^{2}=0.1109 \pm 0.0056
$$

where $\Omega_{\mathrm{DM}}$ is the ratio of the dark matter mass density to the critical density, and $h=0.710 \pm 0.025$ is the scaled Hubble parameter in units of $100 \mathrm{~km} \mathrm{sec}^{-1} \mathrm{Mpc}^{-1}$. The uncertainty will be improved by the PLANCK satellite [4].

In order to calculate the number density of relic particles $\chi$ accurately, in principle one must solve the Boltzmann equation, which describes the evolution of the distribution function. In the standard thermal WIMP production scenario, where decoupling occurs in the radiation-dominated epoch, the particle number density is determined only by the thermal average of the product of the annihilation cross section $\sigma$ and the relative velocity of the annihilating particles $v$. In many cases, the thermally-averaged annihilation cross section can be expanded in a power series: $\langle\sigma v\rangle=a+6 b / x+\cdots$, where $x$ is the ratio of the particle's mass $m_{\chi}$ to the temperature $T$, leading to simple analytic formulas for the final abundance [5, 6], although there are some exceptional cases [7]. The desired cross section for reconciling with the WMAP range is found to be approximately $\langle\sigma v\rangle \sim 3 \times 10^{-26} \mathrm{~cm}^{3} \mathrm{sec}^{-1}$ for the temperature at which WIMPs decouple from the thermal bath. Analytic

methods for the abundance of relic particles have been developed also in various non-standard cosmological scenarios where the relic abundance is increased or decreased due to a low reheat temperature, the late decay of a scalar field, entropy production at late times, modification of the Hubble expansion rate, or their combination [8, 9, 10, 11, 12, 13, 14, 15, 16, 17, 18, 19, 20]. Such analytic approaches enable us to estimate the relic abundance without tedious numerical computations. 
Recently, it was pointed out that the annihilation rate of dark matter particles can be significantly altered by the so-called Sommerfeld enhancement at low velocities [21, 22, 23, 24]. Anomalous excesses of cosmic positrons reported by PAMELA [25], ATIC [26] and FERMI [27] have motivated us to investigate the Sommerfeld effect because the resulting annihilation cross section is extremely boosted for lower velocities [28, 29, 30]. However, it should be also emphasized that the relic abundance can be significantly reduced by the Sommerfeld effect at chemical decoupling [23, 24. There is also a possibility of a change in the relic abundance after kinetic decoupling due to chemical recoupling of the annihilation interactions [31]. (Semi)analytic treatment for relic abundances for the case of Sommerfeld-enhanced $s$-wave annihilations has been discussed [32, 33, 34, 35]. It was shown that to a very good approximation the standard analytic method reproduces numerically computed relic abundances for Sommerfeld-enhanced $s$-wave annihilations in the limit $\alpha / v \gg 1$, where $\alpha$ is a coupling constant between the WIMP and force carrier [33]. However until now, no analytic formula for approximating relic abundances has been discussed for arbitrary $\alpha / v$, even for the case of massless force carriers.

In this paper, we address the relic abundance of non-relativistic particles whose annihilation rate is altered by the Sommerfeld enhancement when the relic particles decouple from the thermal background*. We find highly accurate functions that describes the transition from non-enhanced thermally averaged cross sections to $1 / v$ enhancement not only for $s$-wave but also for $p$-wave annihilations. We then show that the standard analytic method for the final relic abundances provides accuracy to within $1 \%$ even in the range where $1 / v$ approximation does not work. The derived approximate formula is a powerful tool for estimating relic abundances before potential chemical recoupling.

This paper is arranged as follows. In Section 2, we discuss our method for approximating the thermal average of Sommerfeld-enhanced annihilation cross sections. In Section 3, we describe the standard method for deriving the relic density including Sommerfeld enhancement, and compare our analytic results to numerical computations. Section 4 is devoted to our conclusions.

\footnotetext{
*In Ref. 31] the case with massive force carriers is discussed.
} 


\section{Thermally-averaged annihilation cross section}

In this section, we discuss approximated expressions for the thermal average of Sommerfeld-enhanced WIMP annihilation cross sections.

When WIMPs decouple from thermal background, they are non-relativistic. In the absence of force carrier, the annihilation cross section of WIMPs can be expanded with respect to the relative velocity $v$,

$$
\sigma_{0} v=a+b v^{2}+\mathcal{O}\left(v^{4}\right),
$$

where $a, b$ are constants. For $s$-wave annihilation, $a$ gives the dominant contribution to the annihilation of WIMPs. If the $s$-wave contribution is suppressed, $b$ is described by the $p$-wave contribution. When massless force carriers mediate interactions between annihilating particles, the annihilation cross section is enhanced by the factor [36],

$$
S_{l}=\left[\prod_{s=1}^{l}\left(s^{2}+\frac{\alpha^{2}}{v^{2}}\right)\right] \mathrm{e}^{\pi \alpha / v} \frac{\pi \alpha / v}{\sinh (\pi \alpha / v)(l !)^{2}},
$$

for $l$-partial wave. Here, $\alpha$ is a coupling strength. For example, for the case where the annihilation rate of a fermion-antifermion pair is enhanced by scalar boson exchanges, the coupling strength is given by $\alpha=f^{2} /(4 \pi)$, with $f$ being the Yukawa coupling constant. In this paper, we focus on the first two lowest modes,

$$
S_{s}=\frac{2 \pi \alpha / v}{1-\mathrm{e}^{-2 \pi \alpha / v}}, \quad S_{p}=\left(1+\frac{\alpha^{2}}{v^{2}}\right) \frac{2 \pi \alpha / v}{1-\mathrm{e}^{-2 \pi \alpha / v}} .
$$

The case of $\alpha / v \ll 1$ results in the usual non-enhanced annihilation cross sections as $S_{l}$ approaches to unity. In the opposite limit $\alpha / v \gg 1$, the Sommerfeld enhancement factor is simplified down to $S_{s}=2 \pi \alpha / v$ for $s$-wave annihilation, and to $S_{p}=2 \pi \alpha^{3} / v^{3}$ for $p$-wave annihilation. At the leading order of the $v^{2}$ expansion, we can parametrize the Sommerfeld-enhanced annihilation cross sections as

$$
\sigma v= \begin{cases}a S_{s} & (s \text {-wave }) \\ b v^{2} S_{p} & (p \text {-wave }) .\end{cases}
$$

Inclusion of higher order terms is a trivial extension of the procedure we will present.

Let us consider the enhancement of the WIMP annihilation by introducing the boost factor $B=\langle\sigma v\rangle /\left\langle\sigma_{0} v\right\rangle$. The thermal average of the $s$-wave annihilation cross section is given by

$$
\langle\sigma v\rangle=a\left\langle S_{s}\right\rangle=a \frac{x^{3 / 2}}{2 \sqrt{\pi}} \int_{0}^{\infty} \mathrm{d} v v^{2} \mathrm{e}^{-x v^{2} / 4} \frac{2 \pi \alpha / v}{1-\mathrm{e}^{-2 \pi \alpha / v}} .
$$


Introducing further the variables $y \equiv \alpha \sqrt{\pi x}$ and $t \equiv v \sqrt{x} /(2 \sqrt{\pi})$, the boost factor is solely described by $y$,

$$
B_{s}(y)=\left\langle S_{s}\right\rangle=4 \pi y \int_{0}^{\infty} \mathrm{d} t t \mathrm{e}^{-\pi t^{2}} \frac{1}{1-\mathrm{e}^{-y / t}} .
$$

Similarly, for $p$-wave annihilation, we obtain

$$
B_{p}(y)=\frac{x\left\langle v^{2} S_{p}\right\rangle}{6}=\frac{8 \pi^{2} y}{3} \int_{0}^{\infty} \mathrm{d} t t^{3} \mathrm{e}^{-\pi t^{2}}\left(1+\frac{y^{2}}{4 \pi^{2} t^{2}}\right) \frac{1}{1-\mathrm{e}^{-y / t}} .
$$

In the case where the coupling constant $\alpha$ is small or the temperature is high enough to suppress Sommerfeld enhancement, we can expand the boost factor in the Taylor series,

$$
\begin{aligned}
& B_{\text {Taylor }, s}(y)=1+y+\frac{\pi}{6} y^{2}, \\
& B_{\text {Taylor }, p}(y)=1+\frac{2}{3} y+\frac{3+\pi^{2}}{18 \pi} y^{2} .
\end{aligned}
$$

In the opposite limit, where the cross section is enhanced by $1 / v, \mathrm{e}^{-y / t}$ in the denominators of Eqs.(17) and (8) are negligible, leading to

$$
B_{1 / v, s}(y)=2 y, \quad B_{1 / v, p}(y)=\frac{y^{3}}{3 \pi}+\frac{4}{3} y .
$$

As a simple function that connects the two limiting results for $y \rightarrow 0$ and $y \rightarrow \infty$, we propose the following interpolations:

$$
\begin{aligned}
B_{\mathrm{app}, s}(y) & =\frac{1+7 y / 4+3 y^{2} / 2+(3 / 2-\pi / 3) y^{3}}{1+3 y / 4+(3 / 4-\pi / 6) y^{2}} \\
B_{\mathrm{app}, p}(y) & =\frac{1+11 y / 12+(1 /(6 \pi)+1 / 6+\pi / 18) y^{2}+y^{3} /(3 \pi)+y^{4} /(12 \pi)}{1+y / 4}
\end{aligned}
$$

Notice that these choices are not unique. We found that the above expressions are ones of the simplest fitting functions that can reproduce the exact numerical results for the whole range of $y$, as we will see below.

Figure 1 compares various approximations against the exact boost factor. In Fig.1(a) (Fig!1(b)), the exact boost factor $B_{s(p)}$, Eq.(7) (Eq.(8)) (solid line), its Taylor series up to the quadratic order $B_{\text {Taylor,s(p) }}$, Eq.(9) (dashed), $1 / v$ approximation $B_{1 / v, s(p)}$, Eq.(10) (dotted), and our approximation $B_{\text {app }, s(p)}$, Eq.(11) $(+)$, are shown as a function of $y=\alpha \sqrt{\pi x}$. Notice that our approximation $B_{\text {app }, s(p)}$ completely falls together with its exact results. For the case of $s$-wave ( $p$-wave) annihilation, the accuracy of the Taylor expansion Eq.(9) decreases down to $99 \%$ for $y=0.45(y=0.64)$; 


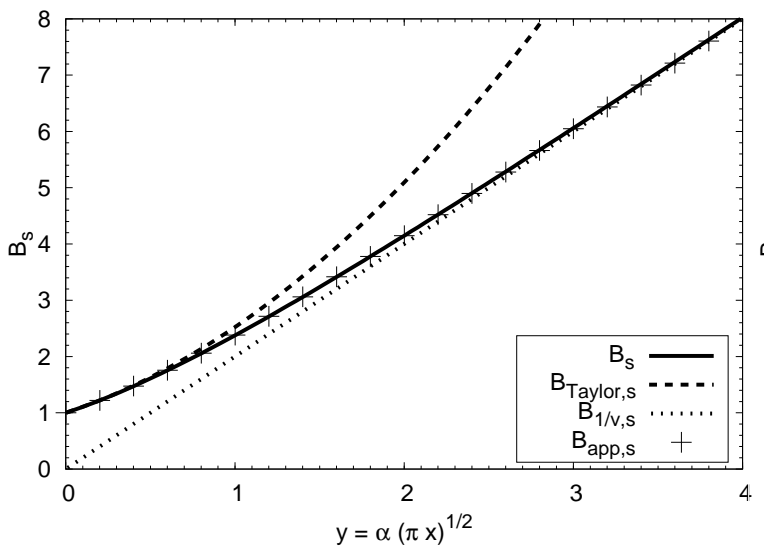

(a)

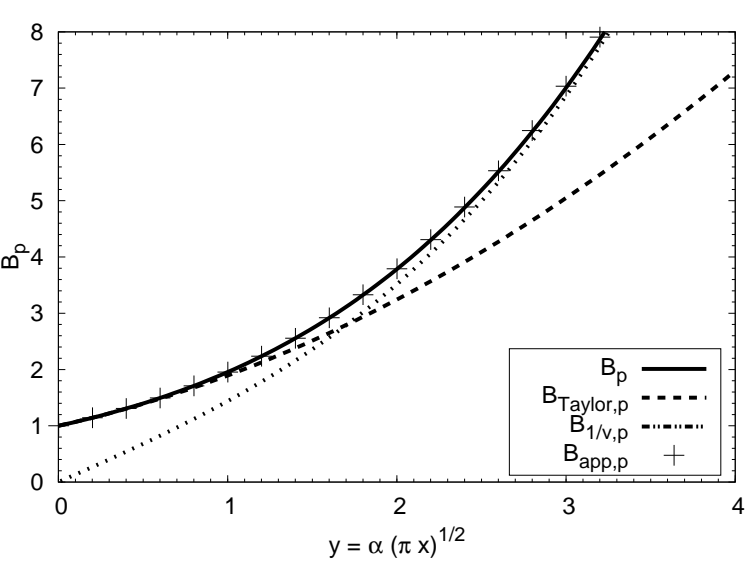

(b)

Figure 1: Exact boost factor $B_{s(p)}$, Eq.(7) (Eq.(8) ) (solid line), its Taylor series up to the quadratic order $B_{\text {Taylor }, s(p)}$, Eq.(9) (dashed), $1 / v$ approximation $B_{1 / v, s(p)}$, Eq.(10) (dotted), and our approximation $B_{\mathrm{app}, s(p)}$, Eq.(11) $(+)$, as a function of $y=\alpha \sqrt{\pi x}$. Our approximation $B_{\mathrm{app}, s(p)}$ completely falls together with its exact results $B_{s(p)}$.

the range for the $1 / v$ approximation Eq.(10) to work at this level is $y>3.0(y>3.6)$. The in-between range $0.45<y<3.0(0.64<y<3.6)$, where neither of the known approximations works, corresponds to $0.06 \lesssim \alpha \lesssim 0.4(0.08 \lesssim \alpha \lesssim 0.5)$ for the typical WIMP decoupling temperature $T \sim m_{\chi} / 20$. On the other hand, our ansatz Eq.(11) always reproduces the exact results with accuracy of less than $0.3 \%(0.9 \%)$.

\section{Relic Abundance with Sommerfeld Enhancement}

Let us discuss the computation of the relic abundance for the case where the annihilation cross section is enhanced by the Sommerfeld factor Eq.(44). As an analytic formalism for the computation of the relic abundance, we follow the standard freezeout picture [1, 5], with appropriate modifications [31, 32, 33, 34, 35]. Here, we show that the standard formalism works even for the case of Sommerfeld enhancement.

The relic density of thermal relic particles $\chi$ whose single production and decay are forbidden by some symmetry is determined by solving the Boltzmann equation,

$$
\frac{\mathrm{d} n_{\chi}}{\mathrm{d} t}+3 H n_{\chi}=-\langle\sigma v\rangle\left(n_{\chi}^{2}-n_{\chi, \mathrm{eq}}^{2}\right)
$$

which describes the time evolution of the number density $n_{\chi}$ of the $\chi$ particles in the universe expanding at the rate $H$. Here $n_{\chi, \text { eq }}$ is the equilibrium value of $n_{\chi}$, whose 
non-relativistic limit is given by

$$
n_{\chi, \mathrm{eq}}=g_{\chi}\left(\frac{m_{\chi} T}{2 \pi}\right)^{3 / 2} \mathrm{e}^{-m_{\chi} / T},
$$

where $g_{\chi}$ denotes the internal degrees of freedom of the $\chi$ particle. At high temperatures, the $\chi$ particles are in thermal equilibrium. After $T$ drops below $m_{\chi}$, the number density $n_{\chi}$ exponentially decreases until the interaction rate $\Gamma=n_{\chi}\langle\sigma v\rangle$ falls below the expansion rate in the radiation-dominated epoch $H=\left(\pi T^{2} / M_{\mathrm{Pl}}\right) \sqrt{g_{*} / 90}$, where $M_{\mathrm{Pl}}=2.4 \times 10^{18} \mathrm{GeV}$ is the reduced Planck mass, and $g_{*}$ the number of the relativistic degrees of freedom. Then, the $\chi$ particles are no longer kept in thermal equilibrium and the comoving number density becomes fixed.

We can express the Boltzmann equation (12) in terms of the dimensionless quantities $Y_{\chi}=n_{\chi} / s$ and $x=m_{\chi} / T$, where $s=\left(2 \pi^{2} / 45\right) g_{*} T^{3}$ is the entropy density. Assuming that the universe expands adiabatically, the Boltzmann equation can be rewritten

$$
\frac{\mathrm{d} Y}{\mathrm{~d} x}=-\frac{4 \pi}{\sqrt{90}} m_{\chi} M_{\mathrm{Pl}} \frac{\sqrt{g_{*}}\langle\sigma v\rangle}{x^{2}}\left(Y_{\chi}^{2}-Y_{\chi, \mathrm{eq}}^{2}\right)
$$

Introducing the variable $\Delta=Y_{\chi}-Y_{\chi, \text { eq }}$, we obtain

$$
\frac{\mathrm{d} \Delta}{\mathrm{d} x}=-\frac{\mathrm{d} Y_{\chi, \mathrm{eq}}}{\mathrm{d} x}-\frac{4 \pi}{\sqrt{90}} m_{\chi} M_{\mathrm{Pl}} \frac{\sqrt{g_{*}}\langle\sigma v\rangle}{x^{2}} \Delta\left(2 Y_{\chi, \mathrm{eq}}+\Delta\right) .
$$

The solution can be analytically derived in two extreme regimes. At temperatures above the freeze-out temperature $T_{F}$, the $\chi$ particles are in thermal equilibrium, so that the deviation of $Y_{\chi}$ from its equilibrium value $Y_{\chi \text {,eq }}$ is very small. Ignoring $\Delta^{2}$ and $\mathrm{d} \Delta / \mathrm{d} x$, the solution is given by

$$
\Delta \simeq \frac{x^{2}}{(8 \pi / \sqrt{90}) m_{\chi} M_{\mathrm{Pl}} \sqrt{g_{*}}\langle\sigma v\rangle},
$$

where we have used $\mathrm{d} Y_{\chi, \text { eq }} / \mathrm{d} x \simeq-Y_{\chi, \text { eq }}$ for $x \gg 1$. Freeze-out occurs when $Y_{\chi}$ deviates from $Y_{\chi, \text { eq }}$ :

$$
\Delta\left(x_{F}\right)=c Y_{\chi, \mathrm{eq}}\left(x_{F}\right) \text {, }
$$

where $x_{F}=m_{\chi} / T_{F}$, and $c$ is a numerical constant of order of unity. Using the early time solution, Eq.(16), we obtain the recursive equation for determining the value of $x_{F}$,

$$
x_{F}=\left.\ln \left(\sqrt{\frac{45}{\pi^{5}}} c m_{\chi} M_{\mathrm{Pl}} g_{\chi} \frac{\langle\sigma v\rangle}{\sqrt{x g_{*}}}\right)\right|_{x=x_{F}}
$$


For the standard $s$ - and $p$-wave annihilation cross sections, the choice of $c=\sqrt{2}-1$ is known to give a good agreement with the numerical results. We will see that this choice is still valid even with Sommerfeld enhancement. At temperatures below $T_{F}$, the production term $Y_{\chi \text {,eq }}$ in the Boltzmann equation can be ignored. Therefore, the final relic abundance is found to be

$$
Y_{\chi}(x \rightarrow \infty)=\frac{1}{(4 \pi / \sqrt{90}) m_{\chi} M_{\mathrm{Pl}} I\left(x_{F}\right)},
$$

where the annihilation integral is defined by

$$
I\left(x_{F}\right)=\int_{x_{F}}^{\infty} \mathrm{d} x \frac{g_{*}\langle\sigma v\rangle}{x^{2}} .
$$

For convenience, we express the final abundance in terms of $\Omega_{\chi} h^{2}=m_{\chi} s_{0} Y_{\chi}(x \rightarrow$ $\infty) h^{2} / \rho_{\text {crit }}$, where $s_{0}=2.9 \times 10^{3} \mathrm{~cm}^{-3}$ is the present entropy density, and $\rho_{\text {crit }}=$ $3 M_{\mathrm{Pl}}^{2} H^{2}$ is the critical density. The conversion factor of the annihilation integral to the relic density is given by

$$
\Omega_{\chi} h^{2}=\frac{8.5 \times 10^{-11}}{I\left(x_{F}\right) \mathrm{GeV}^{2}} .
$$

In sharp contrast to the exact boost factors, Eqs.(7) and (8), our ansatz $B_{\text {app }}$, Eq.(11), leads to simple analytic annihilation integrals. For $s$-wave annihilation,

$$
\begin{aligned}
\frac{I\left(x_{F}\right)}{a \sqrt{g_{*}}}= & \int_{x_{F}}^{\infty} \mathrm{d} x \frac{B_{\mathrm{app}, s}}{x^{2}} \\
= & \frac{1}{x_{F}}+2 \alpha \sqrt{\frac{\pi}{x_{F}}}+\frac{\pi^{2} \alpha^{2}}{6} \ln \left(1+\frac{9 \alpha \sqrt{\pi x_{F}}+12}{(9-2 \pi) \pi \alpha^{2} x_{F}}\right) \\
& +\pi \alpha^{2} \frac{36-11 \pi}{\sqrt{3(117-32 \pi)}}\left(\frac{\pi}{2}-\tan ^{-1} \frac{2(9-2 \pi) \alpha \sqrt{\pi x_{F}}+9}{\sqrt{3(117-32 \pi)}}\right) .
\end{aligned}
$$

For $p$-wave annihilation,

$$
\begin{aligned}
\frac{I\left(x_{F}\right)}{6 b \sqrt{g_{*}}}= & \int_{x_{F}}^{\infty} \mathrm{d} x \frac{B_{\mathrm{app}, p}}{x^{3}} \\
= & \frac{1}{2 x_{F}^{2}}+\frac{4 \alpha \sqrt{\pi}}{9 x_{F}^{3 / 2}}+\frac{\left(3+\pi^{2}\right) \alpha^{2}}{18 x_{F}}+\frac{\left(21-\pi^{2}\right) \sqrt{\pi} \alpha^{3}}{36 \sqrt{x_{F}}} \\
& +\frac{\pi\left(3+\pi^{2}\right) \alpha^{4}}{144} \ln \left(1+\frac{4}{\alpha \sqrt{\pi x_{F}}}\right) .
\end{aligned}
$$

In Fig,2, we show the normalized inverse freeze-out temperature $x_{F}$ as a function of the coupling constant $\alpha$ for the Sommerfeld-enhanced $s$-wave annihilation with 


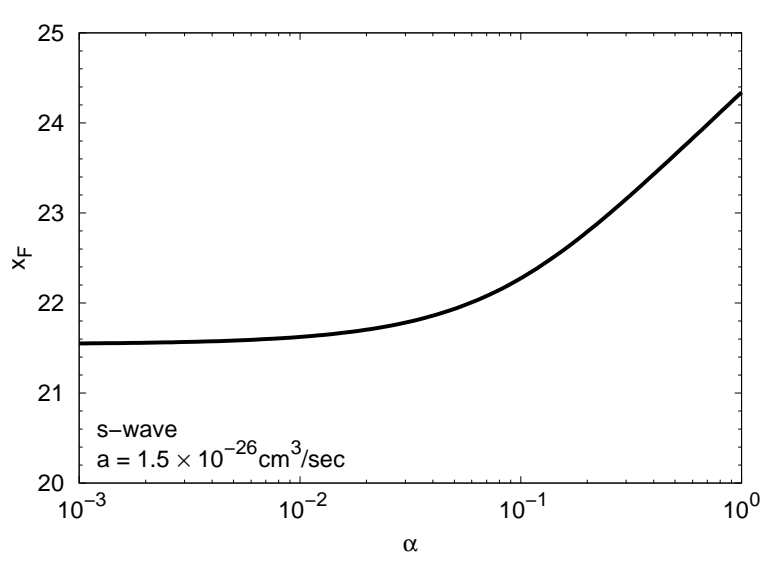

(a)

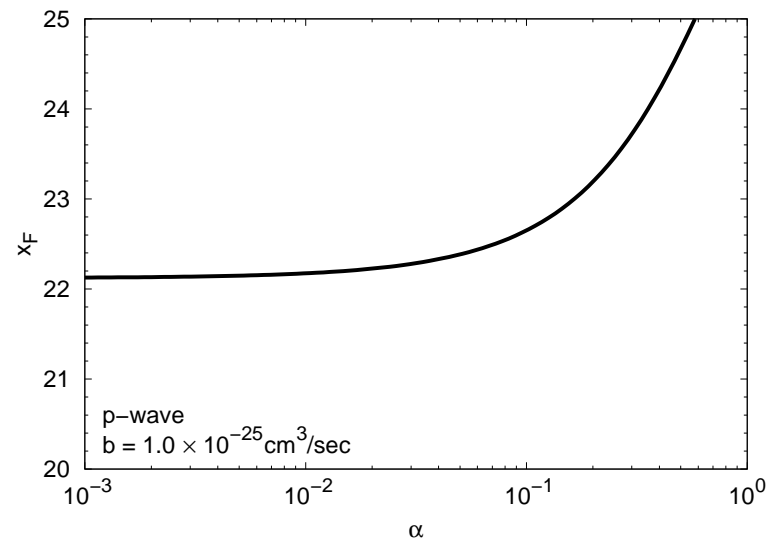

(b)

Figure 2: Normalized inverse freeze-out temperature as a function of $\alpha$ for the Sommerfeldenhanced $s$-wave annihilation with $a=1.5 \times 10^{-26} \mathrm{~cm}^{3} / \mathrm{sec}$ (a), and $p$-wave annihilation with $b=1.0 \times 10^{-25} \mathrm{~cm}^{3} / \mathrm{sec}(\mathrm{b})$. Here we take $m_{\chi}=100 \mathrm{GeV}, g_{\chi}=2$ and $g_{*}=90$.

$a=1.5 \times 10^{-26} \mathrm{~cm}^{3} / \mathrm{sec}(\mathrm{a})$, and $p$-wave annihilation with $b=1.0 \times 10^{-25} \mathrm{~cm}^{3} / \mathrm{sec}$ (b). Here we take $m_{\chi}=100 \mathrm{GeV}, g_{\chi}=2$ and $g_{*}=90$. The plots illustrate that the freeze-out temperature decreases significantly as the coupling constant $\alpha$ increases.

Figure 3(a) compares the analytically estimated relic abundance using Eq.(22) $(+)$ with the numerically computed result (solid line) for the same parameter set as in Fig.2(a). For the $p$-wave case, the approximated relic abundance using Eq.(23) $(+)$ and its exact result (solid) are displayed in Fig.3(b). We also show the relic abundances for $B_{s}=1\left(B_{p}=1\right)$ (dashed) and those for the $1 / v$-enhancement case (dotted). We emphasize that our analytic result successfully describes the transition from the non-enhanced case, $B_{s}=1\left(B_{p}=1\right)$, to $1 / v$-enhancement, and overlaps with the numerically computed results even in the intermediate region $3 \times 10^{-3} \lesssim \alpha \lesssim 0.1$, where neither of the limiting approximations works. In other words, the standard analytic method for the final abundance is also applicable to the Sommerfeld-enhanced annihilation cross section, which exhibits a non-trivial velocity dependence. The deviation of the analytically derived relic abundance from the numerical result is found to be always less than $1 \%$.

\section{Conclusions}

In this paper, we have proposed an approximate analytic function for evaluating the boost factor caused by either $s$ - or $p$-wave Sommerfeld-enhanced annihilation, Eq.(15), 


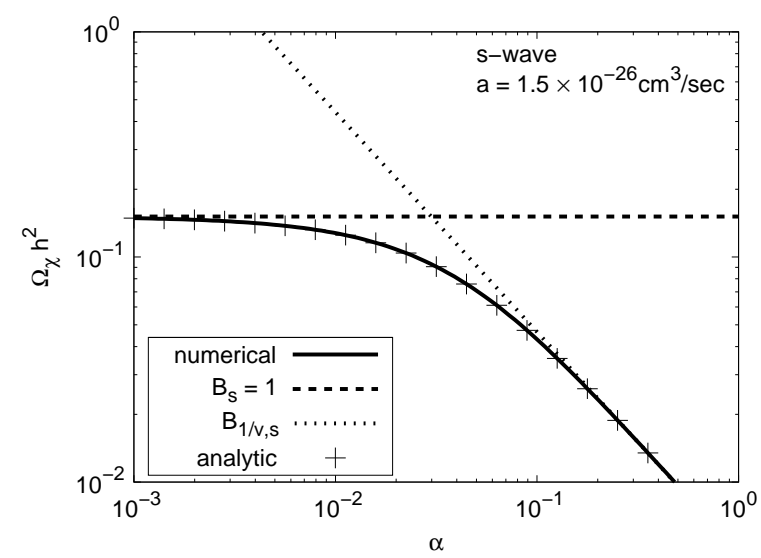

(a)

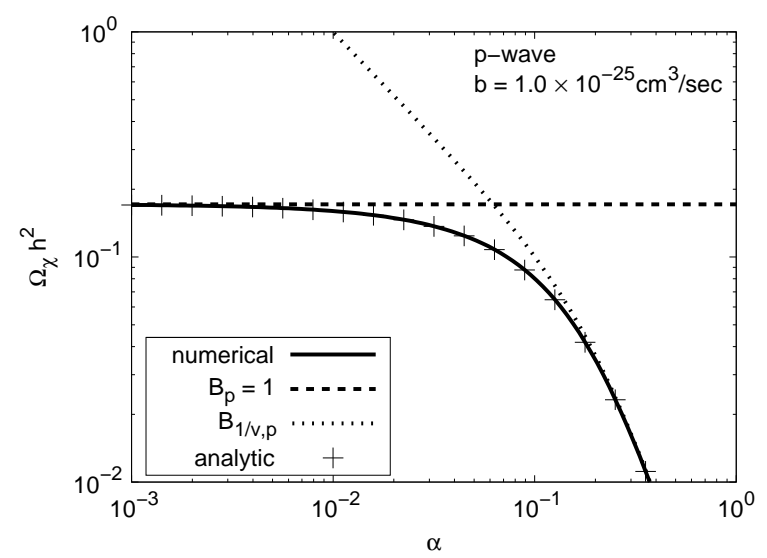

(b)

Figure 3: Comparison of the analytically estimated relic abundance $(+)$ with the numerically computed result (solid line). Here we take the same parameter set as in Fig, The cases of $B_{s}=1$ $\left(B_{p}=1\right)$ (dashed) and $1 / v$-enhancement (dotted) are also shown. Notice that the analytically computed relic abundance using Eq.(22) or (23) $(+)$ completely falls together with the numerical result (solid).

assuming massless force carriers. For $s$-wave ( $p$-wave) annihilation, our ansatz for the boost factor smoothly connects the limiting cases of $B_{s}=1\left(B_{p}=1\right)$ and $1 / v$ enhancement, and reproduces the exact values with accuracy of less than $0.3 \%(0.9 \%)$ in the whole range of the coupling constant $\alpha$. We have applied the approximate boost factor $B_{\mathrm{app}, s}\left(B_{\mathrm{app}, p}\right)$ to the computation of the thermal relic abundance of particles whose $s$-wave ( $p$-wave) annihilation is Sommerfeld-enhanced in the early universe, and derived a totally analytic formula for the final relic abundance in terms of the freeze-out temperature $x_{F}$. Our findings show that the standard analytic method for the relic abundance attains accuracy of less than 1\%. Our results are rather generic, and applicable not only to dark matter candidates but also to other particles that decouple non-relativistically from the thermal background in the early universe.

Finally, we mention that chemical recoupling after kinetic decoupling could affect the relic abundance [31]. A more detailed discussion including the evolution after kinetic decoupling will appear elsewhere.

After completion of this work, we received a preprint [37] that deals with a similar subject. 


\section{Acknowledgements}

The work of M.K. was partially supported by the Marie Curie Training Research "HEPTools" under contract No. MRTN-CT-2006-035505. The work of H.I. is supported by the National Natural Science Foundation of China (11047009) and by the doctor fund BS100108 of Xinjiang university. H.I. also thanks to the hospitality and support of LAPTH, Annecy during the completion of this work.

\section{References}

[1] E. W. Kolb and M. S. Turner, The Early Universe, Addison-Wesley (Redwood City, CA, 1990).

[2] For a review, see G. Bertone, D. Hooper and J. Silk, Phys. Rep. 405, 279 (2005) arXiv:hep-ph/0404175]; G. Jungman, M. kamionkowski, and K. Griest, Phys. Rep. 267, 195 (1996)

[3] WMAP Collab., D. N. Spergel et al., Astrophys. J. Suppl. 148, 175 (2003) arXiv:astro-ph/0302209]; WMAP Collab., D. N. Spergel et al., Astrophys. J. Suppl. 170, 377 (2007) arXiv:astro-ph/0603449]; WMAP Collab., E. Komatsu et al., Astrophys. J. Suppl. 180, 330 (2009) [arXiv:0803.0547[astro-ph]]; WMAP Collab., J. Dunkley et al., Astrophys. J. Suppl. 180, 306 (2009) arXiv:0803.0586 [astro-ph]]; E. Komatsu et al., arXiv:1001.4538 [astro-ph.CO]; N. Jarosik et al., arXiv:1001.4744 [astro-ph.CO].

[4] Planck Science Team, "Planck Bluebook," http://www.rssd.esa.int/planck (2005).

[5] R. J. Scherrer and M. S. Turner, Phys. Rev. D 33, 1585 (1986), Erratum-ibid. D 34, 3263 (1986).

[6] P. Gondolo and G. Gelmini, Nucl. Phys. B 360, 145 (1991).

[7] K. Griest and D. Seckel, Phys. Rev. D 43, 3191 (1991).

[8] R. J. Scherrer and M. S. Turner, Phys. Rev. D 31, 681 (1985).

[9] M. Kamionkowski and M. S. Turner, Phys. Rev. D 42, 3310 (1990).

[10] D. J. H. Chung, E. W. Kolb and A. Riotto, Phys. Rev. D 60, 063504 (1999) arXiv:hep-ph/9809453. 
[11] T. Moroi and L. Randall, Nucl. Phys. B 570, 455 (2000) arXiv:hep-ph/9906527.

[12] G. F. Giudice, E. W. Kolb and A. Riotto, Phys. Rev. D 64, 023508 (2001) arXiv:hep-ph/0005123.

[13] R. Allahverdi and M. Drees, Phys. Rev. Lett. 89, 091302 (2002) arXiv:hep-ph/0203118; Phys. Rev. D 66, 063513 (2002) arXiv:hep-ph/0205246.

[14] P. Salati, Phys. Lett. B 571, 121 (2003) arXiv:astro-ph/0207396.

[15] S. Profumo and P. Ullio, JCAP 0311, 006 (2003) arXiv:hep-ph/0309220.

[16] C. Pallis, Astropart. Phys. 21, 689 (2004) arXiv:hep-ph/0402033.

[17] R. Catena, N. Fornengo, A. Masiero, M. Pietroni and F. Rosati, Phys. Rev. D 70, 063519 (2004) arXiv:astro-ph/0403614.

[18] N. Okada and O. Seto, Phys. Rev. D 70, 083531 (2004) arXiv:hep-ph/0407092.

[19] M. Drees, H. Iminniyaz and M. Kakizaki, Phys. Rev. D 73, 123502 (2006) arXiv:hep-ph/0603165]; Phys. Rev. D 76, 103524 (2007) arXiv:0704.1590 [hepph]]; M. Drees, M. Kakizaki and S. Kulkarni, Phys. Rev. D 80, 043505 (2009) arXiv:0904.3046 [hep-ph]].

[20] A. B. Lahanas, N. E. Mavromatos and D. V. Nanopoulos, Phys. Lett. B 649, 83 (2007) arXiv:hep-ph/0612152.

[21] A. Sommerfeld, Annalen der Physik 403, 257 (1931)

[22] J. Hisano, S. Matsumoto and M. M. Nojiri, Phys. Rev. D 67, 075014 (2003) arXiv:hep-ph/0212022; Phys. Rev. Lett. 92, 031303 (2004) arXiv:hep-ph/0307216; J. Hisano, S. Matsumoto, M. M. Nojiri and O. Saito, Phys. Rev. D 71, 063528 (2005) arXiv:hep-ph/0412403.

[23] J. Hisano, S. Matsumoto, M. Nagai, O. Saito and M. Senami, Phys. Lett. B 646, 34 (2007) arXiv:hep-ph/0610249.

[24] M. Cirelli, A. Strumia and M. Tamburini, Nucl. Phys. B 787, 152 (2007) arXiv:0706.4071 [hep-ph]].

[25] O. Adriani et al. [PAMELA Collaboration], Nature 458, 607 (2009) arXiv:0810.4995 [astro-ph]].

[26] J. Chang et al., Nature 456, 362 (2008). 
[27] A. A. Abdo et al. [The Fermi LAT Collaboration], Phys. Rev. Lett. 102, 181101 (2009) arXiv:0905.0025 [astro-ph.HE]].

[28] M. Cirelli, M. Kadastik, M. Raidal and A. Strumia, Nucl. Phys. B 813, 1 (2009) arXiv:0809.2409 [hep-ph]].

[29] N. Arkani-Hamed, D. P. Finkbeiner, T. R. Slatyer and N. Weiner, Phys. Rev. D. 79015014 (2009) [arXiv:0810.0713 [hep-ph]].

[30] M. Pospelov and A. Ritz, Phys. Lett. B 671, 391 (2009) arXiv:0810.1502 [hep$\mathrm{ph}]]$.

[31] J. L. Feng, M. Kaplinghat and H. B. Yu, arXiv:1005.4678 [hep-ph].

[32] M. Kamionkowski and S. Profumo, Phys. Rev. Lett. 101261301 (2008) arXiv:0810.3233 [astro-ph]].

[33] J. B. Dent, S. Dutta and R. J. Scherrer, Phys. Lett. B 687, 275 (2010) arXiv:0909.4128 [astro-ph.CO]].

[34] J. Zavala, M. Vogelsberger and S. D. M. White, Phys. Rev. D 81, 083502 (2010) arXiv:0910.5221 [astro-ph.CO]].

[35] J. L. Feng, M. Kaplinghat and H. B. Yu, Phys. Rev. Lett. 104, 151301 (2010) arXiv:0911.0422 [hep-ph]].

[36] R. Iengo, JHEP 0905, 024 (2009) arXiv:0902.0688 [hep-ph]]; arXiv:0903.0317 [hep-ph].

[37] S. Hannestad and T. Tram, arXiv:1008.1511 [astro-ph.CO]. 